\title{
MicroRNAs as master regulators of the plant $N B-L R R$ defense gene family via the production of phased, trans-acting siRNAs
}

\author{
Jixian Zhai, ${ }^{1,2}$ Dong-Hoon Jeong, ${ }^{1,2}$ Emanuele De Paoli, ${ }^{1,2,7}$ Sunhee Park, ${ }^{1,2}$ Benjamin D. Rosen, ${ }^{3}$ \\ Yupeng Li, ${ }^{4}$ Alvaro J. González, ${ }^{2}$ Zhe Yan, ${ }^{5}$ Sherry L. Kitto, ${ }^{1}$ Michael A. Grusak, ${ }^{6}$ Scott A. Jackson, ${ }^{4}$ \\ Gary Stacey, ${ }^{5}$ Douglas R. Cook, ${ }^{3}$ Pamela J. Green, ${ }^{1,2}$ D. Janine Sherrier, ${ }^{1,2}$ and Blake C. Meyers ${ }^{1,2,8}$ \\ ${ }^{1}$ Department of Plant and Soil Sciences, University of Delaware, Newark, Delaware 19717, USA; ${ }^{2}$ Delaware Biotechnology \\ Institute, University of Delaware, Newark, Delaware 19711, USA; ${ }^{3}$ Department of Plant Pathology, University of California at \\ Davis, Davis, California 95616, USA; ${ }^{4}$ Institute for Plant Breeding, Genetics, and Genomics, University of Georgia, Athens, \\ Georgia 30602, USA; ${ }^{5}$ Department of Plant Microbiology and Pathology, University of Missouri, Columbia, Missouri 65211, \\ USA; ${ }^{6}$ USDA-ARS Children's Nutrition Research Center, Department of Pediatrics, Baylor College of Medicine, Houston, \\ Texas 77030, USA
}

Legumes and many nonleguminous plants enter symbiotic interactions with microbes, and it is poorly understood how host plants respond to promote beneficial, symbiotic microbial interactions while suppressing those that are deleterious or pathogenic. Trans-acting siRNAs (tasiRNAs) negatively regulate target transcripts and are characterized by siRNAs spaced in 21-nucleotide (nt) "phased" intervals, a pattern formed by DICER-LIKE 4 (DCL4) processing. A search for phased siRNAs (phasiRNAs) found at least 114 Medicago loci, the majority of which were defense-related NB-LRR-encoding genes. We identified three highly abundant 22-nt microRNA (miRNA) families that target conserved domains in these $N B$-LRRs and trigger the production of trans-acting siRNAs. High levels of small RNAs were matched to $>60 \%$ of all $\sim 540$ encoded Medicago NB-LRRs; in the potato, a model for mycorrhizal interactions, phasiRNAs were also produced from NB-LRRs. DCL2 and SGS3 transcripts were also cleaved by these 22-nt miRNAs, generating phasiRNAs, suggesting synchronization between silencing and pathogen defense pathways. In addition, a new example of apparent "two-hit" phasiRNA processing was identified. Our data reveal complex tasiRNA-based regulation of $N B-L R R$ s that potentially evolved to facilitate symbiotic interactions and demonstrate miRNAs as master regulators of a large gene family via the targeting of highly conserved, protein-coding motifs, a new paradigm for miRNA function.

[Keywords: microRNA; tasiRNA; NB-LRR; symbiosis; Medicago]

Supplemental material is available for this article.

Received August 26, 2011; revised version accepted October 20, 2011.

Legume species are agronomically important crops that are rich sources of human dietary protein that also develop unique nitrogen-fixing nodules through a symbiotic relationship with microbes. Root nodules house symbiotic bacteria (rhizobia) that convert atmospheric dinitrogen to ammonia using the energy of the host's photosynthate. During establishment of the symbiosis, rhizobia invade the legume root via a plant-derived

\footnotetext{
${ }^{7}$ Present address: Department of Agriculture and Environmental Sciences, University of Udine, via delle Scienze 208, 33100 Udine, Italy. ${ }^{8}$ Corresponding author.

E-mail meyers@dbi.udel.edu

Article is online at http://www.genesdev.org/cgi/doi/10.1101/gad.177527.111.
}

infection thread and are ultimately released into the host cytoplasm as membrane-bound facultative organelles called "bacteriods," which are the differentiated nitrogen-fixing form or rhizobia. While beneficial interactions with mycorrhiza are known for most plant species (with exceptions that include Arabidopsis), the symbiotic relationships that legume species have with bacteria are highly evolved and require many components related to plant pathogenesis (Deakin and Broughton 2009). Thus, nodulation may require the suppression of host defenses to prevent immune responses; for example, the classically defined, allelic Rj2 and Rfg1 loci from soybeans restrict nodulation with specific rhizobial strains and encode a TIR-NB-LRR (TNL) protein (Yang et al. 2010). 
The only known function of plant NB-LRR proteins is in microbial recognition as activators of defense responses (Eitas and Dangl 2010).The hundreds of diverse NB-LRRs encoded in plant genomes comprise an innate immune system that allows recognition of many pathogens (Meyers et al. 2005).

In the last 10 years, the functions of small RNAs in plants have been extensively explored. These molecules in their mature form are generally 20-24 nucleotides (nt) and are produced by several genetically separable pathways. Plant microRNAs (miRNAs) are typically 21 or 22 nt and function in a post-transcriptional manner by down-regulating target gene products involved in a variety of cellular processes (Bartel 2004; Jones-Rhoades et al. 2006; Mallory and Vaucheret 2006). Another major class of small RNAs are heterochromatic siRNAs (hc-siRNAs) that suppress the activities of transposable elements and maintain genome stability via DNA methylation and chromatin modifications (Vaucheret 2006). Trans-acting siRNAs (tasiRNAs) are a third class of plant small RNAs. Their formation is dependent on miRNA triggers /Allen et al. 2005; Yoshikawa et al. 2005) and requires either the so-called "two-hit" model of dual miRNA target sites in the noncoding RNA precursor (Axtell et al. 2006) or "one-hit" (single-target site) by 22-nt miRNAs (Chen et al. 2010; Cuperus et al. 2010). Four families comprising eight tasiRNA loci have been described in Arabidopsis, while hundreds of noncoding loci of unknown function generate phased small RNA in grasses (Johnson et al. 2009; The International Brachypodium Initiative 2010). The noncoding TAS3 gene is broadly conserved in seed plants (Axtell et al. 2006). Here we report that the model legume Medicago encodes and produces a much more expansive number of phased siRNA (phasiRNAs) and that these are predominantly associated with regulation of numerous and diverse members of the $N B-L R R$ class of disease resistance genes. We demonstrate a substantial network of miRNAs and resulting phasiRNAs that target $N B-L R R$ genes, and we propose that the suppression of the small RNA silencing system and disease resistance machinery may play a role in plant-microbe interactions. While most of these findings were made in Medicago, we present evidence indicating that parallels exist in other legumes and nonleguminous plant species. These data demonstrate that a small number of miRNAs function as master regulators of arguably the largest gene families found in plant genomes.

\section{Results}

miRNA identification in Medicago truncatula and soybeans

Although numerous miRNAs have been identified from legume species, the availability of complete genome sequences provides an opportunity for identification of poorly conserved or other novel miRNAs. In a recent release of miRBase (version 16), a total of 383 miRNA genes have been annotated in M. truncatula, 203 have been annotated in Glycine max (soybean), and many fewer have been annotated in Arachis hypogaea (peanut) and Phaseolus vulgaris (common bean). We believed that miRNA identification is not yet saturated in these species. Therefore, we used a larger set of libraries and tissues, combined with the more complete genome sequences of $M$. truncatula and soybeans, comparative genomics methods, a powerful new miRNA prediction pipeline, and large-scale validation of target cleavage to identify new legume miRNAs, phased or trans-actinglike small RNAs, and hc-siRNAs. Twenty-one small RNA libraries were made from tissues of four legumes, including $M$. truncatula, soybeans, peanuts, and common beans (Supplemental Table S1). These libraries included $\sim 62$ million small RNA reads. Given the absence of peanut and common bean genomes, we used those species' data for comparative analysis and focused on the $M$. truncatula and soybean data.

Using the $M$. truncatula libraries, we applied an informatics pipeline for filtering plant miRNAs from the complete set of small RNAs. Our input to this pipeline was 9,282,720 distinct small RNAs sequenced from eight M. truncatula libraries (Supplemental Fig. S1); among these sequences were 98 different, known, mature $M$. truncatula miRNA sequences of the 383 annotated in miRBase version 16 . The other 285 annotated miRNAs not sequenced may be weakly expressed in the tissues we sampled or are not real miRNAs. After passing the data through the pipeline (see the Supplemental Material), 90 M. truncatula miRNA candidates remained, generated from 137 precursors, which were compared against miRBase version 16 to identify high-similarity homologs. Excluding $26 \mathrm{M}$. truncatula miRNAs that were previously annotated, 22 sequences were found with $>85 \%$ similarity to known plant miRNAs, leaving 42 new miRNA candidates from 51 precursors (Supplemental Table S2). A similar strategy for the soybean genome and small RNA libraries started from 6,133,687 distinct small RNAs and 166 annotated soybean miRNAs and identified 40 new miRNA candidates from 45 precursors (Supplemental Table S3).

We noticed that many $M$. truncatula and soybean miRNA sequences were $22 \mathrm{nt}$ in length, some of which were quite abundant. Recent reports show that 22-nt miRNAs are necessary and sufficient to trigger phasing at tasiRNA loci (Chen et al. 2010; Cuperus et al. 2010). In our M. truncatula data, the 22-nt miR1507, miR1509, miR2109, miR2118, and miR2597 were highly abundant (Supplemental Table S2). Several of these mature miRNAs previously were annotated with sizes other than $22 \mathrm{nt}$, and, based on our libraries, we believe those earlier size annotations are incorrect. For example, in miRBase, miR2109 is annotated as $20 \mathrm{nt}, \operatorname{miR} 2597$ is annotated as $21 \mathrm{nt}$, and miR1509 is annotated as $21 \mathrm{nt}$. In M. truncatula, we predicted eight new 22-nt miRNAs (Supplemental Table S2C), plus we found 22-nt variants of previously described 21-nt miRNAs (miR156 and miR169), and we found 22-mers that passed our miRNA filters, corresponding to the miRNA ${ }^{\star}$ positions of conserved miRNAs (two copies of miR169*, plus miR398*) (Supplemental Table S2B). This abundance of 22-nt miRNAs is in 
contrast to Arabidopsis, which has few 22-nt mature miRNAs annotated in miRBase, most of which occur at low abundances: miR173 (targeting TAS1 and TAS2), miR393, miR472, and miR828 (targeting TAS4). The soybean genome included at least 28 loci producing 22-nt mature miRNAs (Supplemental Table S3). miR1507, miR1509, and miR2118 are highly abundant 22-nt miRNAs in both $M$. truncatula and soybeans (Supplemental Tables S4,S5). Three differences of abundant 22-nt miRNAs were observed in the $M$. truncatula-soybean comparison: miR2597 was abundant in M. truncatula and not found in soybeans, miR1512 was abundant in soybeans but not found in M. truncatula, and miR2109 was abundant in both species but was $22 \mathrm{nt}$ in $M$. truncatula and predominantly $21 \mathrm{nt}$ in soybeans. The conservation and expression of these 22-nt miRNAs in peanuts and common beans are described below. We concluded that 22-nt miRNAs are both numerous and expressed abundantly in legumes.

\section{Identification and validation of $\mathrm{M}$. truncatula miRNA targets}

We predicted potential miRNA targets and integrated the matches with empirical cleaved mRNA data to identify valid miRNA targets. The 90 unique $M$. truncatula miRNA candidate sequences from 137 precursors that passed our filters were searched against both genome and cDNA sequences. Predicted matches with penalty scores $\leq 5(>50,000$ predicted miRNA-target pairs in the genome, and $>15,000$ pairs from cDNAs) were combined with a PARE (parallel analysis of RNA ends) library (German et al. 2008) made from M. truncatula flower tissue (Supplemental Table S1). The observation of several conserved miRNA-target pairs (miR156-SPL, miR172-AP2, miR167-ARF8, and miR390-TAS3) suggested that library quality was sufficient for validation of miRNA targets; these targets exhibited precise, highabundance cleavage products at the predicted target sites (Supplemental Fig. S2).

To detect novel mRNA targets in $M$. truncatula, we used several stringent filters for the PARE data (see the Materials and Methods). We confirmed 144 cleavage sites from 89 genes and 30 intergenic regions, targeted by 46 different miRNAs (Supplemental Table S4A). These targets include a broad set of genes not previously known to be targets of miRNAs. While most target genes were cleaved by only one miRNA at a single recognition site, we identified two target sites for miR1509 in Medtr7g012810; this is significant because in the Arabidopsis TAS3 gene, two "hits" by the 21-nt miR390 are required to trigger the production of tasiRNAs (Axtell et al. 2006). The combination of the stringent filters to identify the miRNA candidates and the conservative methods of experimental validation with the PARE data allowed us to confirm 49 functionally validated $M$. truncatula miRNAs in flower tissue, and thus these were added to miRBase. We also propose to correct several $M$. truncatula miRNAs that we believe were annotated previously with incorrect sizes or mature sequences.
Identification of tasiRNA-like phasiRNA loci in M. truncatula and soybeans

The identification of two target sites for miR1509 in Medtr7g012810 led us to ask how many M. truncatula miRNAs might trigger tasiRNAs. The eight Arabidopsis "TAS" genes generate miRNA-triggered secondary siRNAs in a 21-nt "phased" pattern (Howell et al. 2007). We previously refined and applied a computational approach to evaluate the phasing pattern of small RNAs (De Paoli et al. 2009). The small RNAs identified from this algorithm are phased but do not necessarily function in trans (or even in cis); therefore, we call these phased siRNAs "phasiRNAs." Since tasiRNA-generating loci are TAS genes, we propose that phasiRNA-generating loci are PHAS genes.

We applied this same algorithm to the M. truncatula and soybean genomes for our libraries. Using Arabidopsis as a control, all loci with a phasing score $\geq 15$ in any one library are considered above background (summarized in Supplemental Table S5). PhasiRNAs with only 21-nt intervals were identified from a large number of loci (after removing false positives) (see the Materials and Methods). This set included 112 genes and two intergenic regions in M. truncatula (examples in Fig. 1; Supplemental Table S6) and 26 genes and 15 intergenic regions in soybeans (Supplemental Table S7). MtTAS3 is the only M. truncatula phased locus that has been described previously (Jagadeeswaran et al. 2009), and the remainder are novel PHAS loci that have not been previously described in other species.

We integrated our miRNA lists, target prediction, and the PARE data to identify the triggers for the $M$. truncatula PHAS loci. We were able to identify the miRNA triggers for most PHAS loci (summarized in Table 1; Supplemental Table S6). At least 77 of the $114 \mathrm{M}$. truncatula PHAS loci $(\sim 68 \%)$ are triggered via single cleavage of a 22 -nt miRNA trigger; we call this " $1_{22}$ " for a single-target, 22-nt miRNA trigger event. The majority of these PHAS loci were triggered by a few high-abundance 22-nt miRNAs (miR1507, miR1509, miR2109, and $\operatorname{miR} 2118 \mathrm{a} / \mathrm{b} / \mathrm{c})$. There were just a few exceptions to the predominance of $1_{22}$ PHAS loci. We also identified a novel two-hit $\left(2_{21}\right)$ PHAS locus (the second known example, in addition to TAS3), an AP2-like gene (Medtr2g093060). Consistent with the two-hit model, Medtr2g093060 includes a conserved, cleaved miR172 target site (Aukerman and Sakai 2003), plus a predicted noncleaving and highly degenerate miR156 target site (Fig. 2A). While the miR156 target site has extensive mismatches and a poor score, no other target sites were identified in the upstream region for any other miRNA. The miR172 upstream direction of the phasiRNAs and the validation of the cleaved site in the PARE data resemble TAS3, consistent with the two-hit model of phasiRNA biogenesis from this transcript. Two other related miR172 targets identified in the $M$. truncatula genome (Medtr7g100590 and Medtr4g061200) do not have this miR156 site and thus showed no evidence of phasiRNAs. Soybean orthologs of Medtr2g093060 (Glyma13g40470 and Glyma15g04930, two due to genome duplications) 

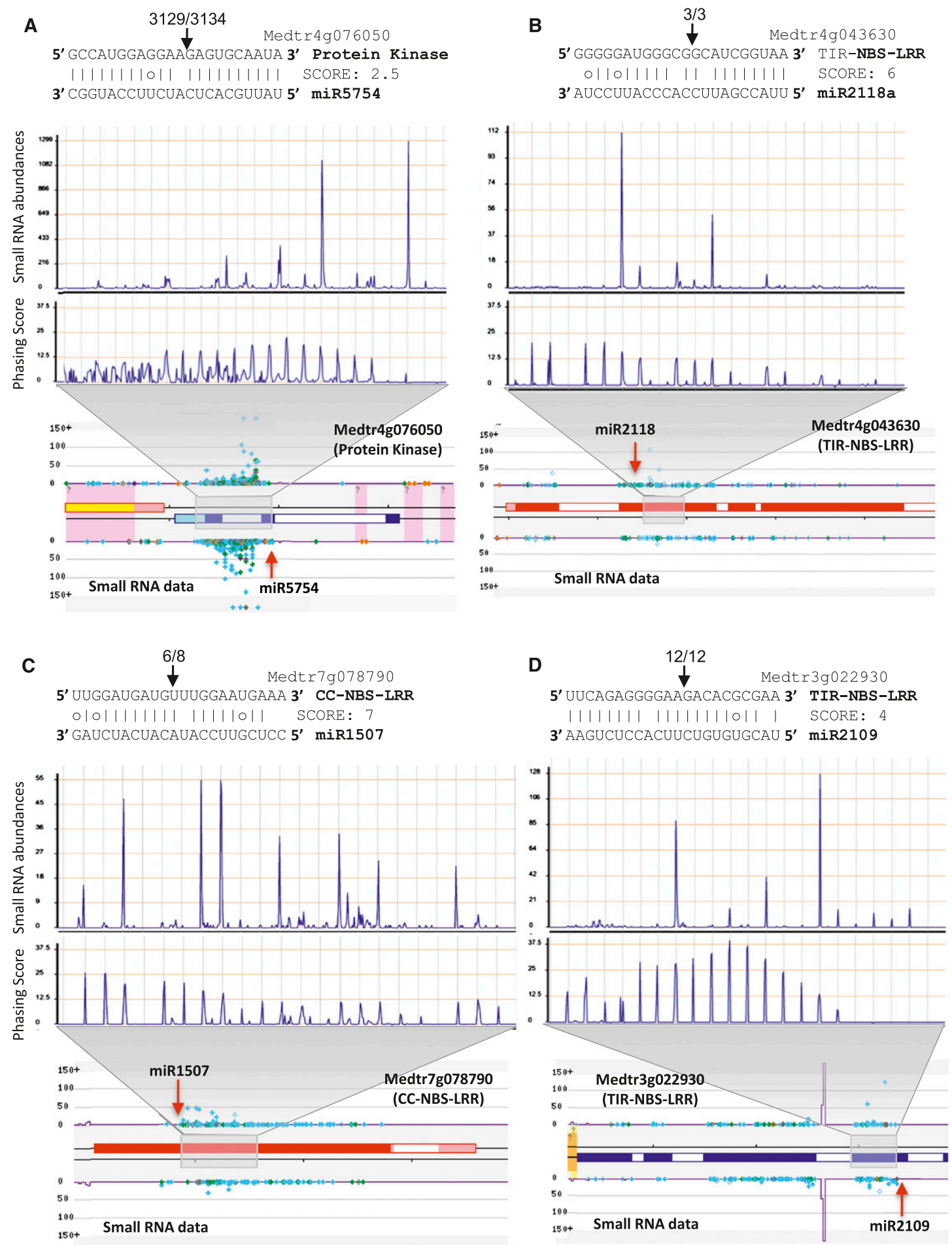

Figure 1. Twenty-two-nucleotide miRNAs trigger phased siRNA production in M. truncatula. Above are alignments of wellconserved miRNAs and their targets; in the alignment, vertical lines indicate matches, missing lines indicate mismatches, and G:U wobble pairs are indicated with a circle. Black arrowheads above the target sequence indicate the cleavage site in the target, and the numbers above separated by the backslash indicate the number of PARE reads in the small window ( $\mathrm{W}_{\mathrm{S}}$ ) (first number) and the number of reads in the large window $\left(\mathrm{W}_{\mathrm{L}}\right)$ (second number), as described in the Supplemental Material. Below the alignments are small RNA abundances and phasing score distributions for the regions indicated by the gray trapezoids; abundances are normalized in TPM (transcripts per million). Below this are images from our Web site showing the M. truncatula flower small RNA data at each PHAS locus, with the red arrowhead pointing to the miRNA cleavage site. Colored spots are small RNAs with abundances indicated on the Y-axis; light-blue spots indicate 21-nt sRNAs, green spots are 22-nt sRNAs, orange spots are 24-nt sRNAs, and other colors are other sizes. Blue boxes (on the bottom strand) or red boxes (on the top strand) are annotated exons. Purple lines indicate a k-mer frequency for repeats; yellow, pink, or orange shading indicates DNA transposons, retrotransposons, or inverted repeats. $(A)$ The new miRNA mtrmiR5754 targets a gene encoding a protein kinase; both the miRNA trigger and the phasiRNAs are specific to the flower library. $(B)$ miR2118 targets a gene encoding a TNL. $(C)$ miR1507 targets a gene encoding a CNL. $(D)$ miR2109 targets a gene encoding a TNL. 
Zhai et al.

Table 1. M. truncatula PHAS loci triggered by legume miRNAs

\begin{tabular}{|c|c|c|c|}
\hline miRNA & $\begin{array}{l}\text { Length } \\
\text { (nt) }\end{array}$ & $\begin{array}{l}\text { Targeted gene } \\
\text { family }\end{array}$ & $\begin{array}{l}\text { Number of PHAS } \\
\text { loci in Medicago }\end{array}$ \\
\hline $\operatorname{miR} 390$ & 21 & TAS3 & 1 \\
\hline miR172 & 21 & AP2-like & 1 \\
\hline $\operatorname{miR} 5754$ & 22 & $\begin{array}{l}\text { Predicted protein } \\
\text { kinase }\end{array}$ & 1 \\
\hline $\operatorname{miR} 2109$ & 22 & $N B-L R R \mathrm{~s}$ & 25 \\
\hline $\mathrm{miR} 2118 \mathrm{a} / \mathrm{b} / \mathrm{c}$ & 22 & $\begin{array}{l}\text { NB-LRRs, } \\
\text { Gma-SGS3, etc. }\end{array}$ & 32 \\
\hline $\operatorname{miR} 1507$ & 22 & NB-LRRs, Mtr-DCL2 & 14 \\
\hline miR1509 & 22 & $\begin{array}{l}\text { Predicted transcription } \\
\text { factor }\end{array}$ & 4 \\
\hline $\operatorname{miR} 1515$ & 22 & Gma-DCL2 & - \\
\hline
\end{tabular}

both have conserved miR172 target sequences but lack any conservation of the untranslated region (UTR) that includes the miR156 site and showed no evidence of phasiRNA production; the miR156 target sequence was also not conserved in Lotus japonicas (data not shown). This unique example of an AP2-like M. truncatula PHAS gene suggests that the spontaneous acquisition of a new miR156 or other yet-to-be-identified 5' noncleaving target site is a recent evolutionary event. Another exception to the $1_{22}$ PHAS loci that predominate was a $2_{22}$ PHAS gene demonstrating double cleavage by a 22-nt miRNA (Fig. 2B); Medtr7g012810 is targeted by miR1509 at two cleaved sites (Supplemental Tables S6, S8). Nearly all small RNAs were found between the two cleaved target sites, with a near absence of small RNAs 3' of the poly-Aproximal target site (Fig. 2C). Small RNAs generated immediately downstream from the $5^{\prime}$ cleavage site, as well as those immediately upstream of the $3^{\prime}$ cleavage site, were perfectly in phase with their respective cleavage sites, consistent with bidirectional processing of the central portion of this $2_{22}$ PHAS locus. In summary, the $M$. truncatula small RNA and PARE data identified many $1_{22}$ loci, two $2_{21}$ loci, and one $2_{22}$ PHAS locus.

Two genes known to be involved in small RNA biogenesis (DICER-LIKE2 [DCL2] and SUPPRESSOR OF GENE SILENCING3 [SGS3]) were also identified as PHAS genes (Supplemental Fig. S3). The DCL2 trigger is predicted to be miR1507 in M. truncatula but miR1515 in soybeans (validated by Li et al. 2010), with the phasiRNAs initiating from different sites in the orthologs, consistent with different miRNA target sites (Supplemental Fig. S3A). PhasiRNAs were identified from the soybean ortholog of SGS3 (Glyma05g33260 [GmSGS3]), but not the three paralogs of SGS3 in M. truncatula (data not shown); GmSGS3 was previously validated as a target of a miR2118 family member (Song et al. 2011). The recruitment of genes involved in phasiRNA biogenesis as sources of phasiRNAs suggests a feedback mechanism reminiscent of the regulation of Arabidopsis AGO1 and DCL1 by miR168 and miR162, respectively (Xie et al. 2003; Vaucheret et al. 2004, 2006).

We identified a novel, potentially noncoding, $1_{22}$ PHAS locus in M. truncatula of particular interest because the phasiRNAs were the most abundant in our data set (10- fold higher than TAS3, the most abundant conserved TAS locus), the PARE sequences matched on both strands, and the phasing score was extremely high (Supplemental Table S6; Supplemental Fig. S4). This intergenic region on Chr. 2 has very weak similarity to the PPR family of genes, suggestive of the Arabidopsis TAS2 locus that targets PPR genes (Montgomery et al. 2008b). TAS2 is triggered by miR173, but the trigger of this $M$. truncatula PHAS gene is a novel 22-nt miRNA candidate at moderate abundances in all of our $M$. truncatula libraries but with no known genomic origin (perhaps due to gaps in the genome). The noncoding precursor and tasiRNAs that initiate cleavage on numerous targets (Supplemental Table S7) are reminiscent of TAS genes; we named this locus PHAS_IGR1. The abundant PARE reads from both strands (Supplemental Fig. S4) are not predicted for RDR6 products, since PARE reads are derived from poly-A mRNA. Another unusual aspect of this locus is the evidence of cleavage by siRNAs acting in cis, also previously reported at TAS3 in which the -D2 siRNA cleaves the primary TAS3 transcript out of phase and without producing secondary siRNAs (Allen et al. 2005; Jagadeeswaran et al. 2009). The cistargeting siRNAs might serve as a negative feedback loop in overall phasiRNA production.

To systematically determine whether the $M$. truncatula phasiRNAs function in cleavage, we again examined the PARE data. By definition, tasiRNAs should function to direct cleavage (or silencing) at second sites (e.g., in trans), although data suggest some may function in cis (Allen et al. 2005). Using the top five phasiRNAs by abundance from each of the 114 PHAS loci and integrating genomic target predictions and our PARE data, a stringent cutoff (see the Materials and Methods) identified $\sim 2000$ sites whose cleavage is guided by these 570 phasiRNAs, including numerous cases of cis regulation (Supplemental Table S8). Therefore, we identified many verifiable trans- and cis-acting siRNAs produced from the large number of legume PHAS loci.

Twenty-two-nucleotide miRNAs as master regulators of legume NB-LRR-encoding genes and generators of phasiRNAs

A feature of the legume phasiRNA loci was the preponderance of NB-LRR-encoding genes, including 79 of $112 \mathrm{M}$. truncatula phasiRNA loci. We call these genes phasi-NB-LRRs, or $p N L s$. We found that just three 22-nt miRNA families (miR1507, miR2109, and miR2118) are responsible for the initiation of the phasiRNAs at 74 of the 79 pNLs (Fig. 3A; Supplemental Table S6). miR1507 "specializes" in targeting CC-NB-LRR (CNL) genes, with strong complementarity to the encoded kinase-2 motif, centered near a highly conserved tryptophan (W) (Fig. 3A). miR2109 targets the TNL class, matching the encoded TIR-1 motif of the TIR domain (described in Meyers et al. 1999). The three-member miR2118 family (miR2118a/b/c; miR2118c is renamed from miR2089) targets sequences encoding the most well-conserved NB-LRR motif, the P-loop (Fig. 3A; Meyers et al. 1999). miR2118a and miR2118c preferentially target $T N L$ genes, while miR $2118 \mathrm{~b}$ almost 
A
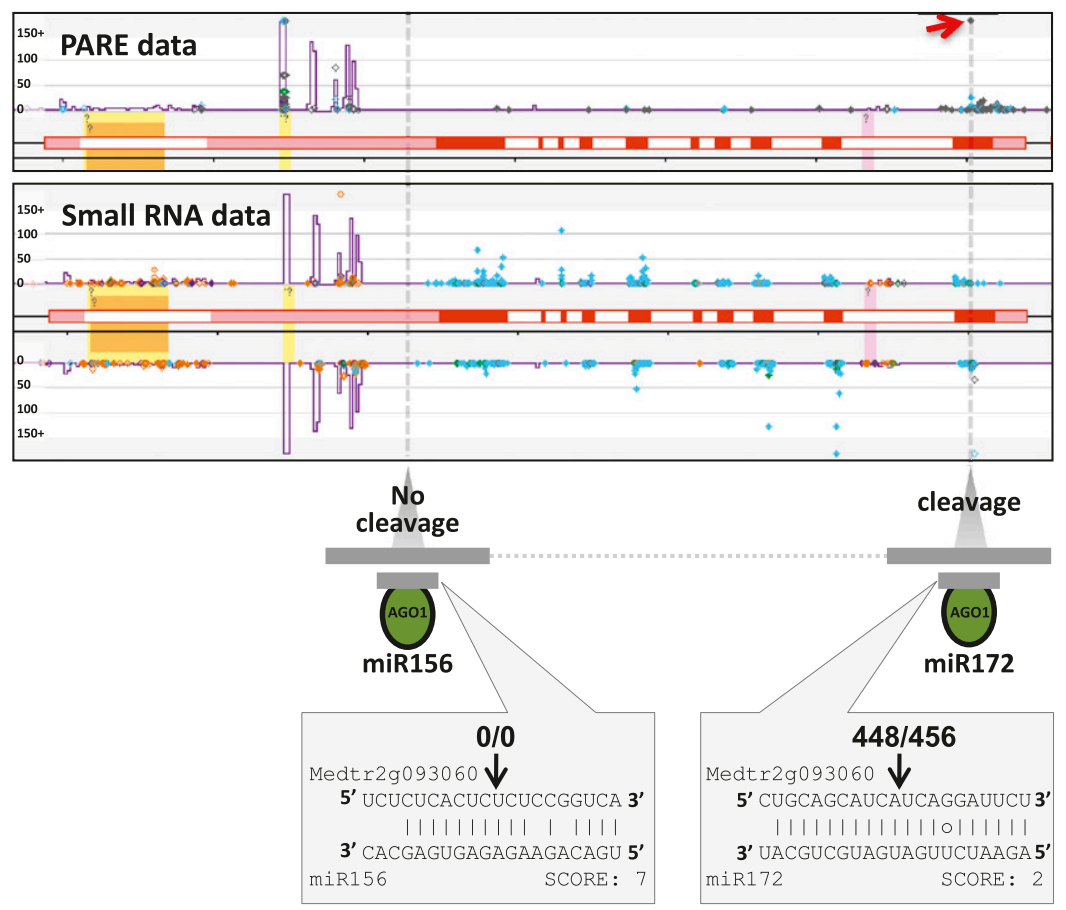

B

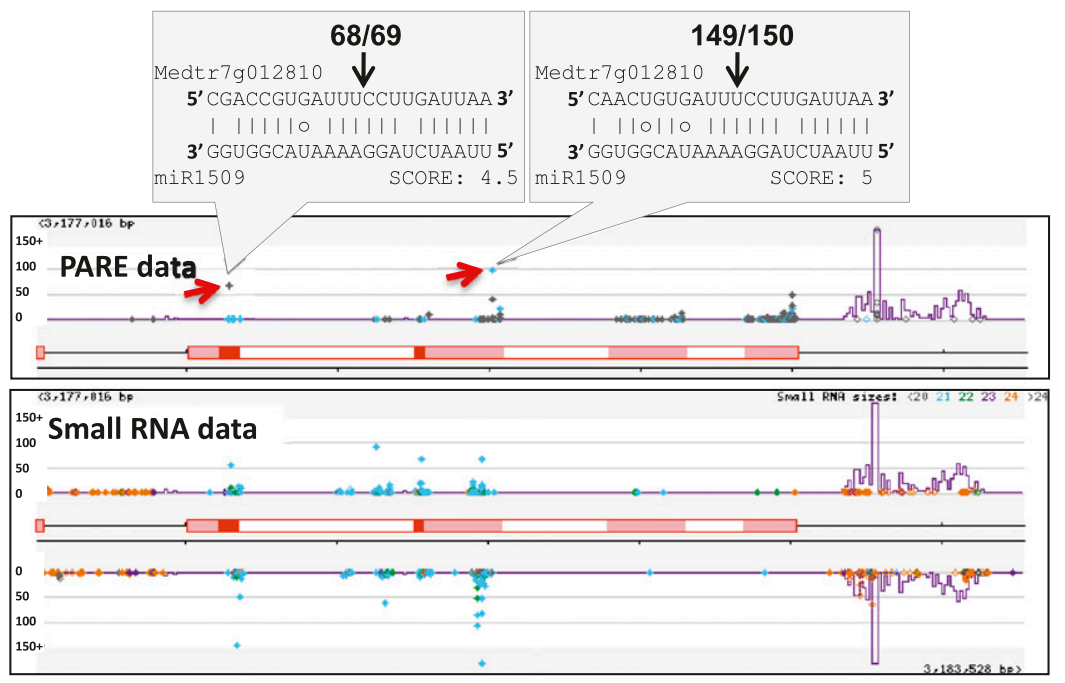

C

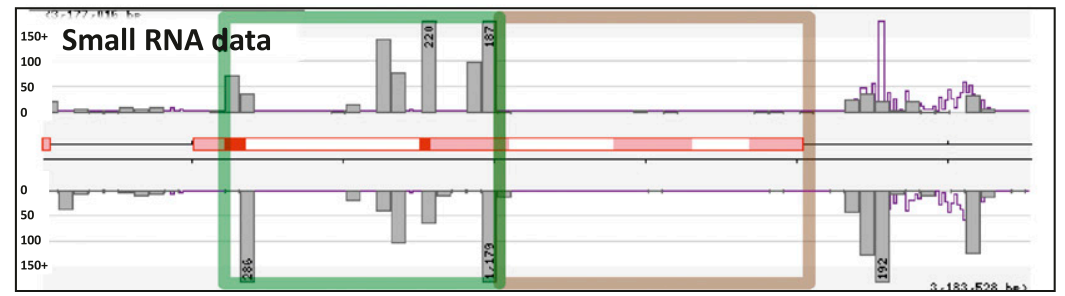

Figure 2. Novel classes of tasiRNAs identified in the $M$. truncatula genome. $(A)$ Example of a $2_{21}$ TAS locus that encodes an AP2 homolog (Medtr2g093060). The top panel shows the PARE data with a highabundance tag from the cleaved site (red arrowhead); for space reasons, only the coding strand data are shown for the PARE tags. The image is interpreted as described in Figure 1. The small RNA data are below; colored dots indicate small RNA sizes, with light blue indicating 21-mers. Other features are as described for the PARE images. The bottom section illustrates the predicted noncleaving miR156 site and the cleaved miR172 site, along with alignments of those miRNAs with the AP2 transcript and the PARE tag abundances. (B) An example of $2_{22}$ TAS locus (Medtr7g012810); double cleavage by the 22-nt miRNA miR1509 triggers phasiRNAs. The first cleavage site occurs on Chr. 7 at nucleotide position 3,178,284; the second is at position $3,180,023$. Both cleavage sites and alignments are indicated, as in $A .(C)$ The small RNA data from the bottom panel of $B$ as a histogram of summed sRNA abundances to emphasize the increased abundance between the two cleavage sites (red arrows in the top panel of $B)$. The green box outlines the region between the two cleavage sites; the brown box indicates the region $3^{\prime}$ of the $3^{\prime}$ 22-nt miRNA cleavage site to the last gene-associated small RNA. exclusively targets $C N L$ genes (Supplemental Table S6). Thus, a specialized group of miRNAs targets conserved domains of $N B-L R R$ s in legumes.

Next, we asked whether the $p N L$ s represent a single, distinct clade within the broader phylogenetic group of $N B-L R R$ s. We mapped the $p N L s$ and their miRNA triggers onto cladograms of the TNL and CNL groups from
M. truncatula (Fig. 3B; Supplemental Fig. S5). pNLs were found to be widely distributed across both the CNL and TNL groups. The triggers for the $p N L s$ were similarly distributed, with no apparent pattern or grouping in the tree; this was especially evident in the TNL group (Fig. 3B). With many small RNAs (>50 TPM [transcxripts per million]) matched to $>60 \%$ of the $\sim 540$ NB-LRRs in the 
A

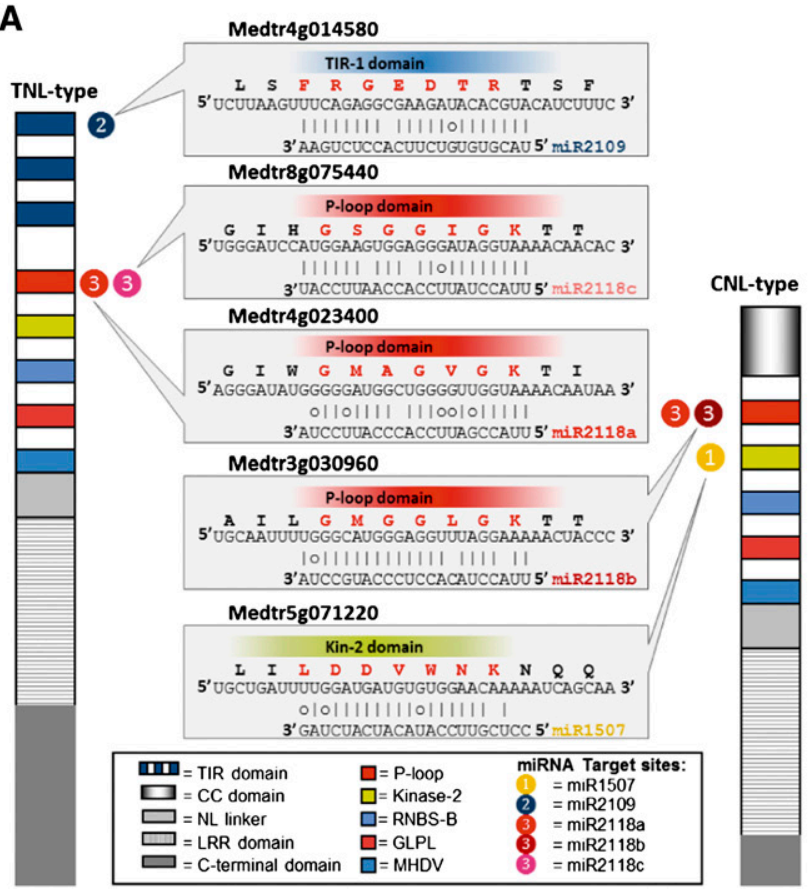

B

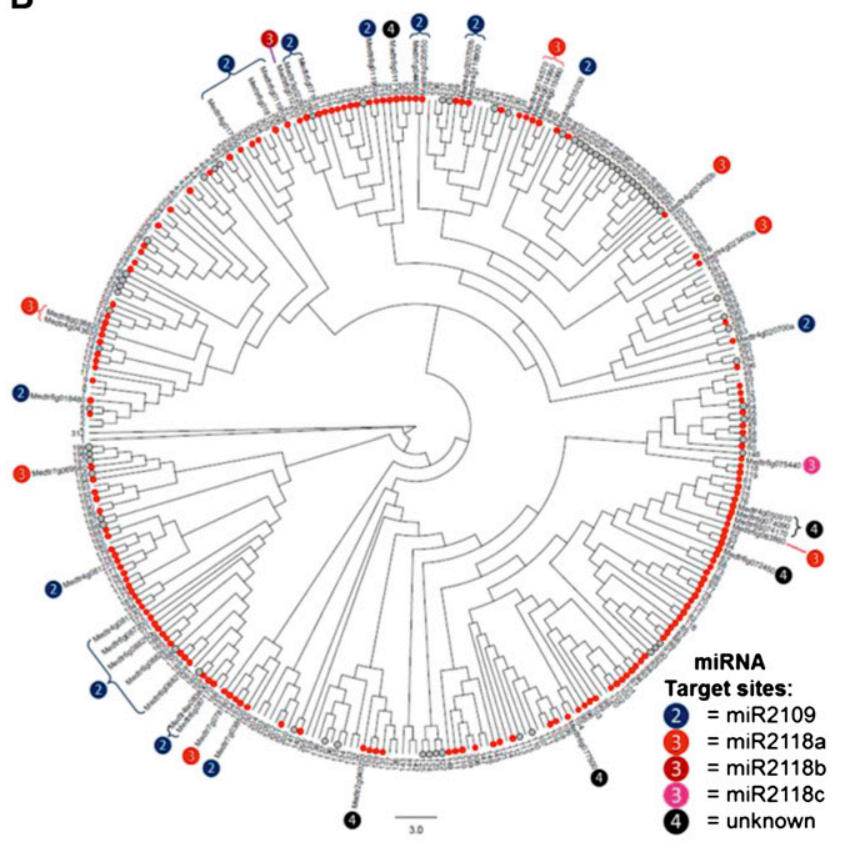

Figure 3. miRNAs trigger phasiRNAs from diverse NB-LRR genes by targeting conserved motifs. (A) Three 22-nt miRNA families, as indicated in the key, trigger the production of phasiRNAs from NB-LRR-encoding genes; miR2118a/b/c are relatively dissimilar members of one family, so all three are shown. On the left and right are illustrations of TNL and CNL proteins, with domains and motifs as indicated in the key at the bottom. Example alignments of target sites for specific miRNAs and motif-encoding regions are indicated in the gray boxes in the center. The alignments are as described in Figure 1, with the exception that the encoded amino acids are marked above the transcript sequence. Red amino acids indicate the core of the conserved motif. $(B)$ An unrooted cladogram was constructed of TNL protein sequences from Medicago. A similar cladogram for the CNLs is found in Supplemental Figure S5. If the gene encoding that protein produces phased small RNAs, the identifying name in the tree was left intact (e.g., Medtr4g050410); the identifier for genes not generating phased small RNAs was replaced by a small integer (e.g., 115). Red dots denote genes with small RNAs exceeding 50 TPM in their summed, hit-normalized abundances. Gray dots denote NB-LRRs from the unassembled Medicago genomic contigs; these were not used in our small RNA analysis. Targets of miRNAs described in the text are marked using symbols as indicated in the key. The miR2118 family is similar enough that it is possible that more than one of these miRNAs targets the same gene, possibly confounding some of our predictions of which miR2118 family member is the trigger. Curly braces group genes targeted by the same miRNA.

assembled Medicago genome (Fig. 3B; Supplemental Fig. S5A; Supplemental Table S9), there may be many more $p N L$ s. An analysis of all NB-LRR-associated small RNAs demonstrated that they were predominantly 21 -mers, and the majority of $N B-L R R$ s have more 21-nt small RNAs than any other size class (Supplemental Fig. S5B,C). This indicates that the silencing of NB-LRRs by phased small RNAs is a phenomenon that occurs broadly across the gene family via a small number of miRNA triggers, each of which is effective against distantly related members of the family. These unique attributes are the result of the targeting of sequences encoding a conserved protein motif by a miRNA, an activity that has not been previously described but may provide flexibility yet rigidity to target a large number of diverse yet related genes.

We next searched for evidence of soybean $p N L s$, identifying 13 pNL loci. Only 13 of 41 soybean PHAS loci are pNLs (Supplemental Table S7). Glyma16g33780 produces limited phased 21-nt siRNAs; this is the Rj2/Rfg1 allelic pair, a TNL of known function that defines rhizobial host specificity (Yang et al. 2010). PhasiRNAs from this gene and many $p N L s$ match more than one gene; those from
$R j 2 / R f g 1$ averaged more than four matching genomic loci. This interconnectivity between $p N L s$ would facilitate an extensive and coordinated regulatory network. In the absence of soybean PARE data, we assessed whether the soybean $p N L$ triggers were related to the $M$. truncatula pNL triggers. The highly expressed $M$. truncatula miR1507 is well conserved in soybeans and is predicted to initiate $p N L$ s (data not shown). The $M$. truncatula 22-nt miR2109 is predominantly $21 \mathrm{nt}$ in soybeans, suggesting that it may not initiate soybean $p N L s$ (vs. triggering $\geq 14$ $p N L s$ in M. truncatula), consistent with fewer $p N L$ in soybeans. In soybeans, the miR2118 family is slightly more complicated, because this miRNA is multicopy and is the star sequence to miR482 (J Zhai and BC Meyers, unpubl.). We checked to see whether the $M$. truncatula and soybean $p N L s$ are found in syntenic locations indicative of an origin early in legume evolution; only two pairs were found in syntenic blocks (Mt-TAS3:Gma-TAS3a and Mt-TAS3:Gma-TAS3b) and no pNLs were syntenic (Supplemental Table S10). This finding and the broad phylogenetic distribution of miRNA targets and $p N L s$ suggest a dynamic nature to the subset of $N B-L R R$ s that are $p N L s$. 
Evolutionary conservation of 22-nt miRNA families from legumes

We examined sequence conservation among 30 diverse and agronomically relevant plant species, including lower plants and basal angiosperms, for homologs of the six most-abundant and PHAS targeting 22-nt legume miRNA families: miR1507, miR1509, miR1510, miR1515, miR2109, and miR2118. The common bean and peanut data demonstrated that most of the 22-nt miRNAs were highly abundant within legumes (Fig. 4; Supplemental Fig. S6). Comparisons across the larger set of species showed these miRNAs present at low abundances (<10 TPM) (yellow in Fig. 4) in many species; those levels are low enough that the precursors and expression will need to be verified when the genomes become available. Analysis of the grape, maize, and potato genomes showed that some but not all of these small RNAs come from predicted miRNA-like hairpins (data not shown). At a more robust threshold of abundance of 11-100 TPM, we found that miR1507, miR1509, miR1515, and miR2118 are present in nonleguminous species (Fig. 4). miR1507 is highly abundant in grapes and avocados, and miR2118 was broadly represented and relatively abundant ( $\geq 100$ TPM) outside of legumes, including moderate signals in all four libraries of the Ginko (GBI in Fig. 4) and Norway spruce (PAB in Fig. 4). This presence in two gymnosperms that date back $>250$ million years suggests that these phased siRNAs may represent an ancient regulatory mechanism.

miR2118 was also abundant in potatoes (Fig. 4), and the potato genome was recently sequenced (Xu et al. 2011), so we examined whether this genome also contains many phasiRNAs or even $p N L s$. At the same cutoff used previously $(\geq 15)$, the three potato small RNA libraries identified 36, 33, and 43 PHAS loci (Supplemental Table S5). Examination of a subset of these phased loci identified numerous $p N L s$ (Supplemental Fig. S7A). Many of these phasiRNAs also matched to $N B-L R R$ s clustered on Chr. 11 (Supplemental Fig. S7B), a region of the chromosome known to contain many active disease resistance genes (Gebhardt and Valkonen 2001). Our analysis of the potato small RNA data suggested that the triggers of these pNLs also include the recently described 22-mer miR5300 (Mohorianu et al. 2011), which targets the encoded P-loop of NB-LRRs within a nucleotide of the same site as miR2118 (Supplemental Fig. S7B). Together with a recent report of pNLs in grapes (http://www.intl-pag.org/19/abstracts/ W84_PAGXIX_525.html), these data suggest that 22-nt $p N L$-targeting miRNAs evolved early in plants and that $p N L$ s are found in nonleguminous species.

\section{Discussion}

We identified a large number of novel miRNAs, and the analysis of these miRNAs and their targets has substantially expanded our understanding of small RNA biology in plants. The extensive network of phasiRNAs is apparently absent in Arabidopsis, yet we believe that the implications of our data go beyond legumes, as we also demonstrated the presence of many PHAS loci and their miRNA triggers in other plants.

\section{Legume miRNAs have evolved in unique ways}

We characterized novel miRNAs in legumes and identified and validated novel legume targets. By integrating PARE data with small RNA data and novel bioinformatics analyses, we identified 42 new miRNA candidates from 51 precursors in $M$. truncatula and 40 new miRNA candidates from 45 precursors for soybeans. Our analysis demonstrated that both the $M$. truncatula and soybean genomes encode a larger set of 22-nt miRNAs than any plant genome described to date. This size class of miRNAs has an innate ability to trigger phased small RNA cascades

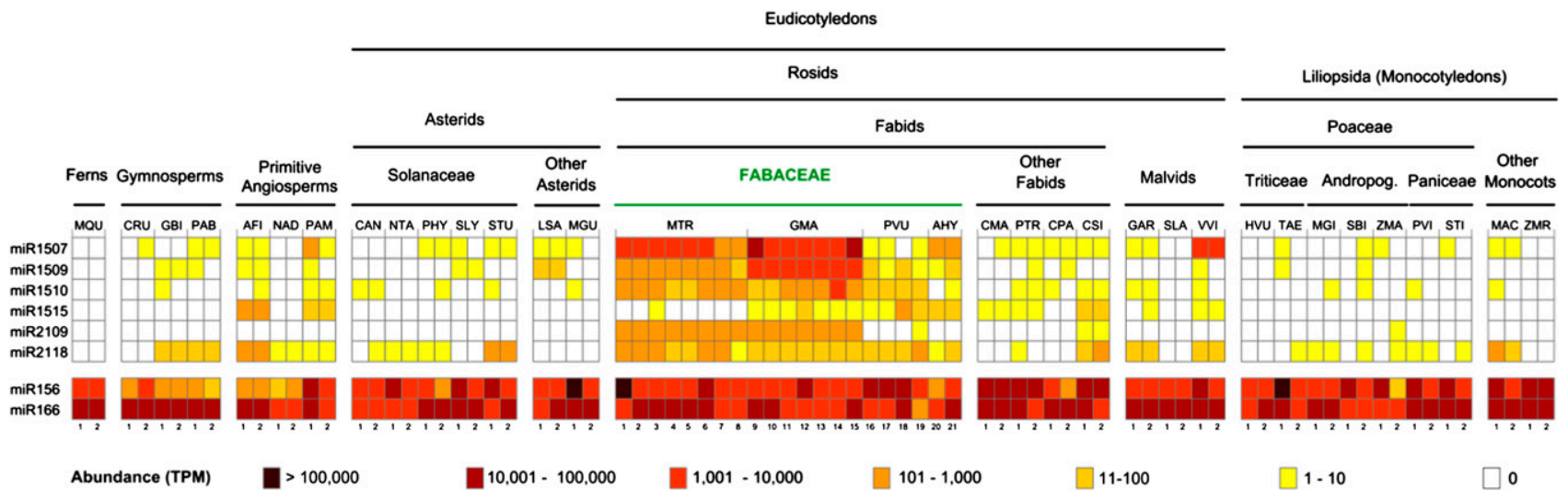

Figure 4. Presence and expression in diverse plant species of 22-nt miRNAs identified from legumes. (Top rows) The presence and abundance of six 22-nt miRNAs that function as phasiRNA triggers were analyzed across 30 species; colors indicate the level of expression in each library, according to the key (shown at the bottom of the figure). The abundance is the sum of all variant sequences, allowing up to three mismatches and two nucleotide shifts at either end. The bottom two rows show highly conserved plant miRNAs (miR156 and miR166) in the same libraries as controls for comparison. Each species is indicated by a three-letter code (codes are defined in the legend to Supplemental Fig. S6), with two columns for each nonlegume species; the first column is a leaf library, and the second column is a flower library, indicated by " 1 " or " 2 " at the bottom of each column. The legume species ("Fabaceae" in green text) are ordered as in Supplemental Table S1. Bars and titles above the species codes indicate the relationships among the species. 
in plants (Chen et al. 2010; Cuperus et al. 2010). The 22-nt miRNAs are produced from at least 21 loci in the $M$. truncatula genome and 28 loci in the soybean genome, whereas Arabidopsis generates just a few known 22-nt mature miRNAs (Cuperus et al. 2010), most of which are weakly expressed. Many of these legume 22-nt miRNAs are highly abundant in the tissues that we characterized. Many of the new 22-nt miRNAs we identified have no or few validated target sites in this set; it is possible that these function to trigger phasiRNAs in tissues we did not examine. The profusion in these legume genomes of 22-nt miRNAs and the phasiRNAs that they initiate suggests that the tasiRNA pathway is more broadly useful as a genetic regulatory circuit, raising the question of whether the relatively low number of phased loci in Arabidopsis is a general feature of plant genomes or is exceptional. This can be addressed by the analysis of phased small RNAs across the increasingly large number of sequenced plant genomes.

Legume phasiRNAs and the general 'rules' for their biogenesis in plants

We identified a large number of loci that fit with the original two-hit (21-nt trigger, or " $22_{21}$ ") or single-hit (22-nt trigger, or " $1_{22}$ ") models for tasiRNA biogenesis (for review, see Allen and Howell 2010); the diversity of phasiRNAs described in our study has implications for understanding the general properties of their biogenesis. For example, we identified a new "two-hit" plant PHAS locus (Fig. 2A), demonstrating that this pathway is not unique to TAS3, a well-conserved but heretofore unique plant developmental regulatory circuit. In fact, evidence of phased siRNAs generated from multiple target sites in a transcript have been reported for the PPR-encoding targets of TAS2 siRNAs and miR161 (Howell et al. 2007; Chen et al. 2010). In our data, the paired configuration of two 21-nt miRNA target sites (cleaving [miR172] and noncleaving [miR156]) is similar to TAS3. Like TAS3, the mRNA fragment $5^{\prime}$ of the miRNA cleavage site in the AP2-like transcript is converted into phased small RNAs, confirming the distinct $5^{\prime}$ directionality of processing under the two-hit model (Axtell et al. 2006). Like miR390, miR172 falls into the minority of conserved plant miRNAs that lack a $5^{\prime} \mathrm{U}$; since the $5^{\prime}$-terminal nucleotide is important for sorting miRNAs and loading onto different Argonaute proteins (Mi et al. 2008), it is possible that this characteristic is functionally important for the two-hit triggers. Montgomery et al. (2008a) demonstrated that miR390 is selectively bound by AGO7, and this AGO7miR390 complex is required at the noncleaving TAS3 target site for tasiRNA biogenesis; they inferred that AGO7 binding may be a requirement for the two-hit tasiRNA pathway. In contrast, miR156, the noncleaving trigger in the $M$. truncatula AP2-like RNA, is known to be bound by AGO1 in Arabidopsis, suggesting that AGO7 loading may not be necessary for two-hit biogenesis of tasiRNAs or that miR156 is bound by AGO7 in M. truncatula.

Our work has expanded our understanding of tasiRNA triggers. This diversity of phasiRNA loci suggests a need for a better organizational scheme to describe these secondary siRNAs. Here we propose to use the term "phasiRNA," as we believe that a tasiRNA cannot be called such without evidence of targeting activity in trans. There is evidence of cis activity in TAS3, and this functional self-targeting small RNA is conserved in Arabidopsis and M. truncatula (Allen et al. 2005; Jagadeeswaran et al. 2009); phasiRNAs targeting their source locus like this could be "casiRNAs." We summarized these classes of phasiRNAs and their precursors in Figure 5A.

In both the two-hit model and a single-hit model for tasiRNA biogenesis, cleavage occurs at only one site; the two "hits" of the two-hit model include one uncleaved target site (Axtell et al. 2006; for review, see Allen and Howell 2010). In our study, a new class was represented by a $2_{22}$ PHAS gene. PhasiRNAs at Medtr7g012810 are triggered after double cleavage by a 22 -nt miRNA. Because 22-nt miRNAs trigger phasiRNA production in the poly-A-proximal fragment of PHAS transcripts, and because we observed high-abundance small RNAs between the two target sites (Fig. 2B,C) yet in phase with both the $5^{\prime}$ cleavage site (downstream phased) and the 3 ' cleavage site (upstream phased), we infer a synergistic effect of having two adjacent 22 -nt target sites. This suggests that a 22 cleavage product is processed inwardly from the two cleavage sites in both the cap- and poly-A-proximal directions, consistent with the directionality of processing for both the single- and two-hit models (Allen and Howell 2010). One alternative explanation for the phasiRNAs at this locus is that the two target sites function independently, whereby we might have expected similar levels of small RNA 3' of both target sites; instead, almost no small RNAs were found $3^{\prime}$ of the $3^{\prime}$-most target site (brown box in Fig. 2C), suggesting that this 22-nt target site does not function as an independent $1_{22}$ site. Based on the conservation of miR1509 across legumes, $2_{22}$-based PHAS genes are likely moderately conserved.

Taken together, these results allow us to refine earlier models for phasiRNA biogenesis. Prior work demonstrated that phasiRNA production is dependent on either $2_{21}$ or $1_{22}$ targeting, resulting in either cap- or poly-Aproximal processing (Fig. 5B; Allen et al. 2005; Axtell et al. 2006; Montgomery et al. 2008b; Chen et al. 2010; Cuperus et al. 2010). We summarized the loci that exemplify these cases in Figure 5C. Experiments have demonstrated that the noncleaving site both is conserved in flowering plants (Axtell et al. 2006) and cannot be replaced by a cleaving site in the $2_{21}$ model (Montgomery et al. 2008a; Felippes and Weigel 2009), although double cleavage has been observed in mosses (Axtell et al. 2006). We showed that $2_{22}$ loci are processed into phasiRNAs almost exclusively between two cleaved target sites (Fig. $2 B, C)$. These data suggest two points: First, the two-hit $2_{22}$ pathway is epistatic to the hypostatic single-hit $1_{22}$ mode of processing; and second, double cleavage can strictly delimit the boundaries of phasiRNA production. Although we do not know the directionality of the processing, if the 22-nt miRNAs trigger $2_{22}$ phasiRNAs, as in the $1_{22}$ PHAS transcripts, processing may occur from both cleaved ends toward the center of the double-cleaved transcript. 
A

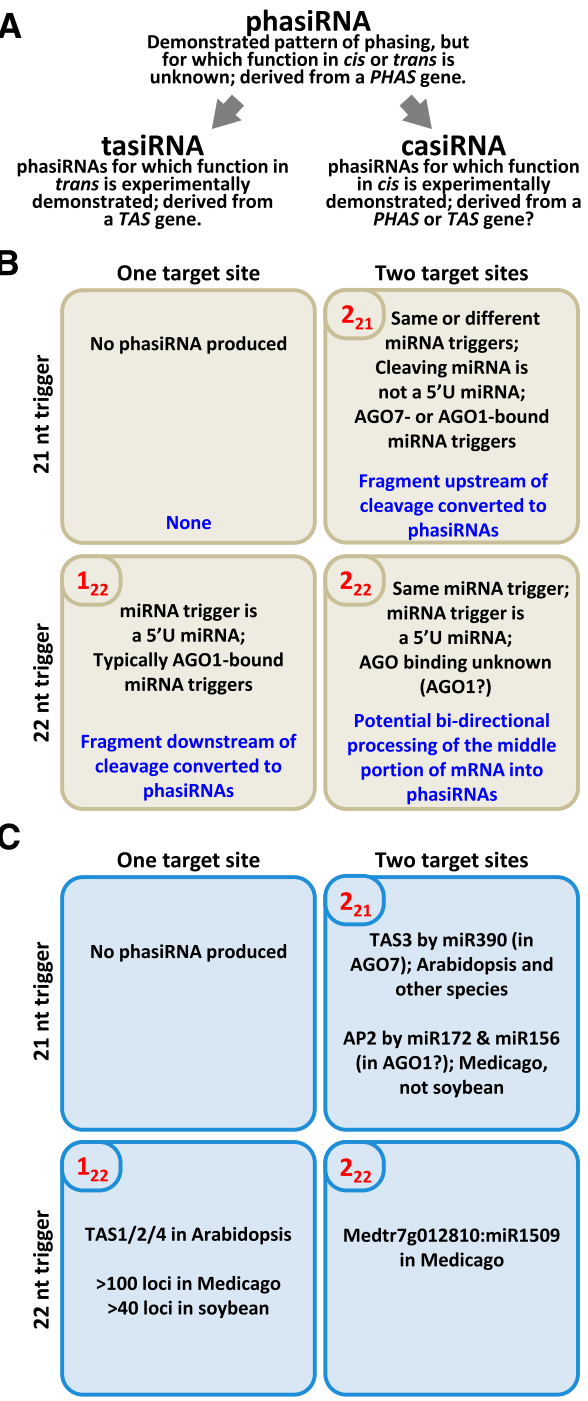

Figure 5. Model of miRNA triggers and target sites of plant phasiRNA biogenesis. (A) Definition of phased small RNA classes in plants. PhasiRNA-generating loci are called PHAS genes, as tasiRNA-generating loci are TAS genes. $(B)$ Rules and observations of phasiRNAs in plants. The two top cells correspond to PHAS genes with 21-nt miRNA triggers, and the two bottom cells have 22-nt triggers; the left cells have one miRNAbinding site, and the right cells have two binding sites. In each of the four cells, black text in boxes is observations of tasiRNAs previously described in Arabidopsis and in this study; blue text indicates which portion of the cleaved TAS transcript is converted to tasiRNAs. In the top left corner of each cell is indicated the name we ascribed to each class; there is no evidence of $1_{21}$ phasiRNAs. $(C)$ Examples of phasiRNAs matching the observations described in $B$.

\section{NB-LRRs are targets of an extensive small RNA regulatory network}

Our study demonstrated that NB-LRRs are targeted by multiple, independent miRNA families, and each of these miRNAs targets a region encoding highly conserved protein motifs. At least three miRNA families in legumes are predicted to target transcripts from hundreds of NB-LRRencoding genes, and phased small RNAs are generated from at least $79 M$. truncatula $p N L$ genes. The 22-mer $p N L$ trigger miR2109 in $M$. truncatula is mostly $21 \mathrm{nt}$ in soybeans; size diversification by altering the proportion of 21-nt versus 22-nt variants of a miRNA may allow flexibility in the degree of silencing of target genes due to differences in their ability to trigger phasiRNAs that amplify post-transcriptional silencing. The larger family of plant $N B-L R R$ targeting miRNAs includes the 22-nt miR472, which was first identified in poplar (S Lu et al. 2007) and later found in Arabidopsis (called miR772 at that time) (Lu et al. 2006), in which it was demonstrated to target and cleave $N B-L R R$ transcripts at P-loop-encoding regions. Based on a simple sequence comparison, both miR472 and miR $1510^{\star}$ are closely related to miR482 and miR2118. The 21-nt miR1510 annotated in soybeans and abundant in our $M$. truncatula libraries is also predicted to target NB-LRR-encoding transcripts (Valdes-Lopez et al. 2010). Most plant miRNAs target many fewer genes than the number of targets of the $p N L$ triggers; this suggests that the $p N L$-triggering miRNAs are also quite unusual because they target conserved motifs, regulating an extensive gene family. In the 1990s, degenerate oligos were widely used to amplify large sets of NB-LRRs (Michelmore 1996); it now appears that nature beat scientists to the punch, taking advantage of the "degeneracy" of miRNA-target interactions to broadly interact with the NB-LRR gene family.

$p N L$ triggering may evolve rapidly. The $p N L s$ are distributed throughout the CNL and TNL families of $M$. truncatula and are well represented in soybeans. We found no evidence for synteny among pNLs in these two legume genomes. One interpretation of the lack of synteny between the $M$. truncatula and soybean pNLs is that miRNA target sites may be gained or lost with relative ease by substitutions in the few nucleotides for which changes would not disrupt the protein motif but would disrupt the miRNA-mRNA interaction. While deeper sequencing may yet identify paralogous legume $p N L s$, the $p N L$ subset of $N B-L R R$ s may evolve relatively quickly depending on microbial selection pressures.

Is this phenomenon of $p N L s$ specific to the legumes? In Arabidopsis, only one $N B-L R R$, a $T N L$, was identified as generating phasiRNAs (Howell et al. 2007); our reanalysis of this locus with much deeper data did not confirm this, yet there are many 21-nt siRNAs at this locus (J Zhai and BC Meyers, unpubl.) reported to be RDR6-dependent (Howell et al. 2007). Klevebring et al. (2009) described phasiRNAs associated with a small number of $N B-L R R$ s in poplars, but the triggers were not identified. Our crossspecies analysis of the $p N L$-triggering miRNAs demonstrated that at least the miR2118 family (including miR472, miR482, and miR2089) is well conserved in many plant species. miR2118 is particularly interesting because it also triggers phasiRNAs from intergenic regions in rice panicles (Johnson et al. 2009); these are noncoding loci that have no apparent relationship to the miR2118 pNL targets in legumes that we have described.

$p N L s$ potentially function to coregulate en masse the $N B-L R R$ family in legumes. Thus, the small set of $p N L$ triggers could function as master regulators of genes that are the first line of plant defense against many pathogens. 
The extent of this system in legumes makes it tempting to speculate that this is a critical regulatory circuit that is important for symbiosis. There are data to support this idea; for example, overexpression of the 22-nt miR482 (a member of the miR2118 family) leads to hypernodulation in soybeans ( $\mathrm{Li}$ et al. 2010). Li et al. (2010) also demonstrated that miR482 is up-regulated $6 \mathrm{~d}$ after Bradyrhizobium japonicum inoculation, and the $p N L$ trigger miR1507 is up-regulated in a hypernodulating soybean mutant. Differences in the utilization of $p N L$ s between $M$. truncatula and soybeans could potentially reflect well-described differences in their nodulation processes. However, the finding that some of these $p N L$ targeting miRNAs are conserved outside of legumes suggests hypotheses other than a role specific to nodulation; one related possibility would be in the global regulation of $N B-L R R$ genes to promote mycorrhizal colonization, a symbiotic interaction in which Arabidopsis does not participate but that is common among legumes and nonleguminous plants and is particularly well studied in potatoes (Hata et al. 2010). This $p N L$ mechanism could have been refined in legumes for rhizobial symbiosis.

Howell et al. (2007) described the role of phasiRNAs in a gene family $(P P R-P)$ that shares some features with the $N B-L R R$ gene family: It is large and dynamic, targeted by more than one miRNA, and may benefit evolutionarily from diversity in the gene family. Howell et al. (2007) suggest that the phasiRNAs could minimize the number of active $P P R$ copies to suppress gene dosage. $p N L s$ may be part of a similar regulatory system to constrain the number of active $N B-L R R$ s. It has been known for many years that the $T N L$ class of $N B-L R R$ s is absent from grass genomes but is found in lower plants (Meyers et al. 1999). We believe that we have identified a phenomenon distinct from the phasiRNA-generating $P P R$ genes because the $p N L$ siRNAs are generated by direct targeting of large numbers of diverse genes by just a few miRNAs that target several distinct, highly conserved, protein motifencoding sequences. One enduring question has been how an entire clade of genes could be lost or driven out of a genome. Curiously, the miR2118 family is conserved as a phasiRNA trigger between grasses and legumes, but in legumes it targets $N B-L R R$ s and in grasses it targets noncoding RNAs found in intergenic regions that form clusters (Johnson et al. 2009). Perhaps the reason for the absence of the TNL class from grass genomes is because of phasiRNA suppression of the gene family followed by pseudogenization and, ultimately, neofunctionalization as regulatory noncoding RNAs in grasses, although no function is yet known for the grass phasiRNAs. Extending this hypothesis, perhaps the extensive set of noncoding phasiRNA precursors found in grass genomes is derived from the protein-coding TNL family as pseudogenized, extinct PHAS loci that are vestiges of the extant clusters of $p N L s$ observed in the potato genome, for example (Supplemental Fig. S7), which resembles the clustered distribution of those noncoding phasiRNAs loci in grass; such a scenario has been described for the Xist noncoding RNA that functions in X-chromosome inactivation in animals (Duret et al. 2006).
Diverse pathways in legumes are subject to regulation by phased small RNAs

In addition to the $p N L$ genes, we identified a number of protein-coding genes and miRNA triggers that generate phasiRNAs (Table 1). Among the more unusual is an AP2like gene with both a miR156 and a conserved miR172 site; miR156 and miR172 are intimately involved in juvenile-to-adult-phase transition in Arabidopsis (Wang et al. 2009; Wu et al. 2009). However, in Arabidopsis, miR156 targets the SPL family upstream of the miR172targeted AP2-like genes, and the levels of miR156 and miR172 are inversely proportional during maturation of the plant (Wu et al. 2009). The co-occurrence of these miRNA target sites in an AP2-like gene suggests a model in which the $A P 2$ phasiRNAs functions in cis to positively regulate silencing of the AP2 gene (as casiRNAs), while also functioning in trans to target other AP2-like genes.

We also characterized phasiRNA biogenesis from genes important for small RNA biogenesis and pathogen defense. GmSGS3 is targeted by miR2118, the same miRNAs that target many $N B$ - $L R R$ genes. SGS3 has an important role in juvenile development (Peragine et al. 2004) and is critical in tasiRNA production (Elmayan et al. 2009). The silencing of both NB-LRR genes and SGS3 by miR2118 suggests a coupling of these regulatory events. DCL2 genes in both $M$. truncatula and soybeans independently acquired 22-nt miRNA target sites, resulting in phasiRNAs from this gene in both species (Supplemental Fig. S3A); interestingly, overexpression of miR1515, the soybean DCL2 trigger, was demonstrated to lead to hypernodulation (Li et al. 2010). Target analysis of the M. truncatula DCL2 suggests that the phasiRNA trigger is miR1507, the $C N L$-specific $p N L$ trigger. Because DCL2 is important for small RNA biogenesis and SGS3 is important for tasiRNA biogenesis, these results are reminiscent of the DCL1miR162 (Xie et al. 2003) or the AGO1-miR168 feedback loops (Vaucheret et al. 2004, 2006). Since DCL2 and SGS3 are involved in both small RNA silencing and viral resistance (Mourrain et al. 2000), and we demonstrated a substantial network of miRNAs and resulting phasiRNAs that target $N B-L R R$ genes, we propose that the suppression of the small RNA silencing system and disease resistance machinery may play a role in plant-microbe interactions. Therefore, our data are indicative of an extensive regulatory network controlling the transcript levels in these interactions. While most of these findings were made in $M$. truncatula, we have evidence indicating that parallels exist in other legumes and nonleguminous species, suggesting a small number of miRNAs function as master regulators of the largest gene families found in plant genomes.

\section{Materials and methods}

\section{Plant materials}

M. truncatula $A 17$ (Jemalong) For developing seeds, flowers from greenhouse-grown plants were date-tagged at full bloom, and pods were selected for seed collection at $20 \mathrm{~d}$ after anthesis. For seedlings, seeds were surface-sterilized and sown on water agar in the dark as in Catalano et al. (2004), with whole seedlings 
collected $24 \mathrm{~h}$ post-sowing. Foliage, roots, and flowers were collected from plants grown aeroponically with complete nutrient supplementation within a controlled environmental chamber at $55 \%$ relative humidity and a $14-\mathrm{h}, 22^{\circ} \mathrm{C}$ day/ $10 \mathrm{~h}, 18^{\circ} \mathrm{C}$ night cycle. Foliage and roots were collected 3 wk post-sowing, and flowers were collected at $-1,0,+1$ tripping. For nodules, plants were grown aeroponically for 1 wk with $1 / 2 \times$ nutrient solution, transferred to nitrogen-free medium for $7 \mathrm{~d}$, and inoculated with Sinorhizobium meliloti strain 2011 (Meade et al. 1982) $10^{6}$-CFU (colony-forming unit) plant-1 to induce nodule formation in a method modified from Catalano et al. (2004). Nodules of mixed developmental ages were collected at 14 d post-inoculation. Root knots were collected from an established root culture maintained on $1 / 2$ MS salts, $20 \mathrm{~g} / \mathrm{L}$ sucrose, $0.5 \mathrm{mg} / \mathrm{L}$ nicotinic acid, $0.5 \mathrm{mg} / \mathrm{L}$ pyridoxine $\cdot \mathrm{HCl}, 0.4 \mathrm{mg} / \mathrm{L}$ thiamine. $\mathrm{HCl}$, and $8 \mathrm{~g} / \mathrm{L}$ Phytagar (GIBCO) after incubation in the dark for $6 \mathrm{wk}$ post-inoculation at $28^{\circ} \mathrm{C}$ with sterile Meloidogyne incognita eggs. Medicago/Glomus intraradices colonized and mock-inoculated roots were grown according to Liu et al. (2007).

G. max Williams 82 samples were collected as in Joshi et al. (2010).

P. vulgaris "Bat 93 " seeds and flowers were collected from greenhouse-grown plants as for Medicago. Foliage and nodules were collected from aeroponically grown plants by the same method as Medicago, except that nodule formation was induced by the addition of Rhizobium leguminosarum bv. viciae 3841, and nodules were collected and pooled at 7,14 , and $21 \mathrm{~d}$ postinoculation.

A. hypogaea were grown in greenhouse conditions and induced to form nodules as in VandenBosch et al. (1994). Nodules and foliage were collected at 7,14, $21 \mathrm{~d}$ post-inoculation with B. japonicum NC92.

\section{Sequencing of small RNAs from legumes}

Total RNA was isolated using Trizol reagents or plant RNA reagent, both from Invitrogen. $M$. truncatula small RNA libraries (except MTR01) were constructed and sequenced at Illumina; other libraries were constructed as previously described (C Lu et al. 2007) and sequenced on an Illumina GAIIx instrument at the Delaware Biotechnology Institute.

Twenty-one small RNA libraries were made from the materials described above, representing four legume species, including eight libraries from Medicago, seven from G. max (soybean), two from $A$. hypogaea (peanut), and four from $P$. vulgaris (common bean). Approximately 62 million small RNA sequences were obtained after removing adapters and low-quality reads, with trimmed lengths between 18 and $34 \mathrm{nt}$. After excluding small RNAs matching structural RNAs $/ \mathrm{t} / \mathrm{rRNA}$ loci), 12.1 million and 12.6 million reads were mapped to the Medicago genome (Mt3.5) (http://www.medicago.org) and the G. max genome (Gmax101) (Schmutz et al. 2010), respectively. Consistent with previous reports of plant small RNAs, small RNAs in all legume tissues are predominantly found in two sizes: $21 \mathrm{nt}$ and 24 nt (Supplemental Fig. S8; Supplemental Table S11). We developed two Web sites and databases, using the Medicago and soybean genomes, to store and analyze all 21 libraries; we used these visualization tools extensively for this analysis. These Web sites are available at http://mpss.udel.edu/mt_sbs and http:// mpss.udel.edu/soy_private.

\section{Small RNA informatics analysis}

The miRNA prediction pipeline is outlined in Supplemental Figure S1, with details of the filters explained in the Supplemental Material.
We used CleaveLand to predict miRNA targets (Addo-Quaye et al. 2009). An astringent filter retained all matches with scores $\leq 5$; scoring was assigned by CleaveLand, and described previously (Allen et al. 2005). The PARE data were integrated using the pipeline described in the Supplemental Material.

Phasing analysis was performed as described previously (De Paoli et al. 2009). As a final check of loci with phasing scores $\geq 15$, scores and abundances of small RNAs from each high-scoring locus were graphed and checked visually to remove false positives such as miRNAs with numerous low-abundance peaks that could incorrectly pass our filters. We also manually removed unannotated tRNA and rRNA-like loci with high phasing scores because of their high small RNA levels.

\section{Comparative analysis of miRNAs from diverse plant species}

We used libraries from 30 diverse species plus the legume libraries. The sequences of the eight miRNAs of interest were searched for exact and near matches, allowing three mismatches and up to a 2-nt shift at either the $5^{\prime}$ or $3^{\prime}$ end or both. Small RNAs 20-24 nt in size and represented by at least two reads in a library were analyzed. Alignments were performed using SeqMap (Pawlowski et al. 2004) followed by output filtering and reformatting by custom-written PERL scripts. Heat maps were created using customized PERL scripts and the Inkscape vector graphics software (http://www.inkscape.org).

Synteny analysis of the M. truncatula and soybean PHAS loci

To identify orthologous pairs of PHAS loci in the Medicago (Mt3.5) and soybean (Gmax101) genomes, we performed pairwise alignments by BLASTN with default parameters. Pairs of sequences with high similarity (identity $>60 \%$ and aligned coverage $>500 \mathrm{bp}$ ) were selected to check for synteny between Medicago and soybeans by comparing their locations to syntenic blocks between Medicago and soybeans detected by SyMAP (Soderlund et al. 2011). Because of the possibility that some small regions of colinearity may have been masked by a larger region, MUMMer (Kurtz et al. 2004) was used to align each pair of 1-Mb extended regions (500 kb on each side of each PHAS locus) in order to further identify sequences that are in the region of colinearity.

Phylogenetic analysis of the family of NB-LRR proteins encoded in the M. truncatula genome

We extracted predicted protein sequences for 312 TNLs and 385 CNLs from the Medicago genome sequence, including unassembled contigs. The unassembled contigs were not integrated into the genome, and thus were not used for phased siRNA or any small RNA analysis. A total of 542 NB-LRR proteins were encoded in the assembled Medicago genome and used for small RNA analysis; the subset of these NB-LRRs with a high level of small RNAs ( $\geq 50$ TPM) is denoted in the cladograms with red dots. The remaining 155 NB-LRRs presumably map to gaps in the assembled genome sequence; these are denoted in the cladograms by gray dots.

The cladograms were constructed using maximum parsimony based on only the nucleotide-binding site of the NB-LRR proteins (the conserved NB-ARC domain). Separate TNL class and CNL class trees were rooted with the nearest neighbor in the other class determined from a joint tree (data not shown).

\section{GenBank accession numbers}

The GenBank Gene Expression Omnibus (GEO) accession numbers for these data are GSE28755 for the small RNA data 
from 30 diverse plants (also found at http://smallrna.udel.edu) and GSE31061 for the legume small RNAs and PARE sequencing results. The legume data are also available at http:// mpss.udel.edu/mt_sbs and http://mpss.udel.edu/soy_sbs.

\section{Acknowledgments}

We thank the M. truncatula genome consortium (N. Young and colleagues), S. Knapp, and M. Harrison for plant materials, and M. Nakano for informatics. This work was supported primarily by USDA award 2006-03567 (to D.J.S., B.C.M., and P.J.G.), plus NSF award 0638525 (to B.C.M. and P.J.G.), NSF award 0701745 (to B.C.M.), NSF award 0605251 (to D.R.C.), NSF award IOS1025752 (to G.S.), and funds from USDA-ARS under agreement number 58-6250-0-008 (to M.A.G.).

\section{References}

Addo-Quaye C, Miller W, Axtell MJ. 2009. CleaveLand: A pipeline for using degradome data to find cleaved small RNA targets. Bioinformatics 25: 130-131.

Allen E, Howell MD. 2010. miRNAs in the biogenesis of transacting siRNAs in higher plants. Semin Cell Dev Biol 21: 798804.

Allen E, Xie Z, Gustafson AM, Carrington JC. 2005. MicroRNAdirected phasing during trans-acting siRNA biogenesis in plants. Cell 121: 207-221.

Aukerman MJ, Sakai H. 2003. Regulation of flowering time and floral organ identity by a microRNA and its APETALA2-like target genes. Plant Cell 15: 2730-2741.

Axtell MJ, Jan C, Rajagopalan R, Bartel DP. 2006. A two-hit trigger for siRNA biogenesis in plants. Cell 127: 565-577.

Bartel DP. 2004. MicroRNAs: Genomics, biogenesis, mechanism, and function. Cell 116: 281-297.

Catalano CM, Lane WS, Sherrier DJ. 2004. Biochemical characterization of symbiosome membrane proteins from Medicago truncatula root nodules. Electrophoresis 25: 519-531.

Chen HM, Chen LT, Patel K, Li YH, Baulcombe DC, Wu SH. 2010. From the cover: 22-nucleotide RNAs trigger secondary siRNA biogenesis in plants. Proc Natl Acad Sci 107: 1526915274.

Cuperus JT, Carbonell A, Fahlgren N, Garcia-Ruiz H, Burke RT, Takeda A, Sullivan CM, Gilbert SD, Montgomery TA, Carrington JC. 2010. Unique functionality of 22-nt miRNAs in triggering RDR6-dependent siRNA biogenesis from target transcripts in Arabidopsis. Nat Struct Mol Biol 17: 997-1003.

Deakin WJ, Broughton WJ. 2009. Symbiotic use of pathogenic strategies: Rhizobial protein secretion systems. Nat Rev Microbiol 7: 312-320.

De Paoli E, Dorantes-Acosta A, Zhai J, Accerbi M, Jeong DH, Park S, Meyers BC, Jorgensen RA, Green PJ. 2009. Distinct extremely abundant siRNAs associated with cosuppression in petunia. RNA 15: 1965-1970.

Duret L, Chureau C, Samain S, Weissenbach J, Avner P. 2006. The Xist RNA gene evolved in eutherians by pseudogenization of a protein-coding gene. Science 312: 1653-1655.

Eitas TK, Dangl JL. 2010. NB-LRR proteins: Pairs, pieces, perception, partners, and pathways. Curr Opin Plant Biol 13: $472-477$.

Elmayan T, Adenot X, Gissot L, Lauressergues D, Gy I, Vaucheret H. 2009. A neomorphic sgs3 allele stabilizing miRNA cleavage products reveals that SGS3 acts as a homodimer. FEBS $J$ 276: $835-844$.

Felippes FF, Weigel D. 2009. Triggering the formation of tasiRNAs in Arabidopsis thaliana: The role of microRNA miR173. EMBO Rep 10: 264-270.
Gebhardt C, Valkonen JP. 2001. Organization of genes controlling disease resistance in the potato genome. Annu Rev Phytopathol 39: 79-102.

German MA, Pillay M, Jeong DH, Hetawal A, Luo S, Janardhanan P, Kannan V, Rymarquis LA, Nobuta K, German R, et al. 2008. Global identification of microRNA-target RNA pairs by parallel analysis of RNA ends. Nat Biotechnol 26: 941946.

Hata S, Kobae Y, Banba M. 2010. Interactions between plants and arbuscular mycorrhizal fungi. Int Rev Cel Mol Bio 281: 1-48.

Howell MD, Fahlgren N, Chapman EJ, Cumbie JS, Sullivan CM, Givan SA, Kasschau KD, Carrington JC. 2007. Genome-wide analysis of the RNA-DEPENDENT RNA POLYMERASE6/ DICER-LIKE4 pathway in Arabidopsis reveals dependency on miRNA- and tasiRNA-directed targeting. Plant Cell 19: 926-942.

The International Brachypodium Initiative. 2010. Genome sequencing and analysis of the model grass Brachypodium distachyon. Nature 463: 763-768.

Jagadeeswaran G, Zheng Y, Li YF, Shukla LI, Matts J, Hoyt P, Macmil SL, Wiley GB, Roe BA, Zhang W, et al. 2009. Cloning and characterization of small RNAs from Medicago truncatula reveals four novel legume-specific microRNA families. New Phytol 184: 85-98.

Johnson C, Kasprzewska A, Tennessen K, Fernandes J, Nan GL, Walbot V, Sundaresan V, Vance V, Bowman LH. 2009. Clusters and superclusters of phased small RNAs in the developing inflorescence of rice. Genome Res 19: 1429-1440.

Jones-Rhoades MW, Bartel DP, Bartel B. 2006. MicroRNAS and their regulatory roles in plants. Annu Rev Plant Biol 57: 19-53.

Joshi T, Yan Z, Libault M, Jeong DH, Park S, Green PJ, Sherrier DJ, Farmer A, May G, Meyers BC, et al. 2010. Prediction of novel miRNAs and associated target genes in Glycine max. BMC Bioinformatics 11: S14. doi: 10.1186/1471-2105-11-S1-S14.

Klevebring D, Street NR, Fahlgren N, Kasschau KD, Carrington JC, Lundeberg J, Jansson S. 2009. Genome-wide profiling of populus small RNAs. BMC Genomics 10: 620. doi: 10.1186/ 1471-2164-10-620.

Kurtz S, Phillippy A, Delcher AL, Smoot M, Shumway M, Antonescu C, Salzberg SL. 2004. Versatile and open software for comparing large genomes. Genome Biol 5: R12. doi: 10.1186/gb-2004-5-2-r12.

Li H, Deng Y, Wu T, Subramanian S, Yu O. 2010. Misexpression of miR482, miR1512, and miR1515 increases soybean nodulation. Plant Physiol 153: 1759-1770.

Liu J, Maldonado-Mendoza I, Lopez-Meyer M, Cheung F, Town CD, Harrison MJ. 2007. Arbuscular mycorrhizal symbiosis is accompanied by local and systemic alterations in gene expression and an increase in disease resistance in the shoots. Plant J 50: 529-544.

Lu C, Kulkarni K, Souret FF, MuthuValliappan R, Tej SS, Poethig RS, Henderson IR, Jacobsen SE, Wang W, Green PJ et al. 2006. MicroRNAs and other small RNAs enriched in the Arabidopsis RNA-dependent RNA polymerase- 2 mutant. Genome Res 16: 1276-1288.

Lu C, Meyers BC, Green PJ. 2007. Construction of small RNA cDNA libraries for deep sequencing. Methods 43: 110-117.

Lu S, Sun YH, Amerson H, Chiang VL. 2007. MicroRNAs in loblolly pine (Pinus taeda L.) and their association with fusiform rust gall development. Plant J 51: 1077-1098.

Mallory AC, Vaucheret H. 2006. Functions of microRNAs and related small RNAs in plants. Nat Genet 38: S31-S36. doi: $10.1038 /$ ng 1791 .

Meade HM, Long SR, Ruvkun GB, Brown SE, Ausubel FM. 1982. Physical and genetic characterization of symbiotic and 
auxotrophic mutants of Rhizobium meliloti induced by transposon Tn5 mutagenesis. J Bacteriol 149: 114-122.

Meyers BC, Dickerman AW, Michelmore RW, Sivaramakrishnan S, Sobral BW, Young ND. 1999. Plant disease resistance genes encode members of an ancient and diverse protein family within the nucleotide-binding superfamily. Plant $J$ 20: 317332.

Meyers BC, Kaushik S, Nandety RS. 2005. Evolving disease resistance genes. Curr Opin Plant Biol 8: 129-134.

Mi S, Cai T, Hu Y, Chen Y, Hodges E, Ni F, Wu L, Li S, Zhou H, Long C, et al. 2008. Sorting of small RNAs into Arabidopsis argonaute complexes is directed by the $5^{\prime}$ terminal nucleotide. Cell 133: 116-127.

Michelmore R. 1996. Flood warning-resistance genes unleashed. Nat Genet 14: 376-378.

Mohorianu I, Schwach F, Jing R, Lopez-Gomollon S, Moxon S, Szittya G, Sorefan K, Moulton V, Dalmay T. 2011. Profiling of short RNAs during fleshy fruit development reveals stagespecific sRNAome expression patterns. Plant J 67: 232-246.

Montgomery TA, Howell MD, Cuperus JT, Li D, Hansen JE, Alexander AL, Chapman EJ, Fahlgren N, Allen E, Carrington JC. 2008a. Specificity of ARGONAUTE7-miR390 interaction and dual functionality in TAS3 trans-acting siRNA formation. Cell 133: 128-141.

Montgomery TA, Yoo SI, Fahlgren N, Gilbert SD, Howell MD, Sullivan CM, Alexander A, Nguyen G, Allen E, Ahn JH, et al. 2008b. AGO1-miR173 complex initiates phased siRNA formation in plants. Proc Natl Acad Sci 105: 20055-20062.

Mourrain P, Beclin C, Elmayan T, Feuerbach F, Godon C, Morel JB, Jouette D, Lacombe AM, Nikic S, Picault N, et al. 2000. Arabidopsis SGS2 and SGS3 genes are required for posttranscriptional gene silencing and natural virus resistance. Cell 101: 533-542.

Pawlowski WP, Golubovskaya IN, Timofejeva L, Meeley RB, Sheridan WF, Cande WZ. 2004. Coordination of meiotic recombination, pairing, and synapsis by PHS1. Science 303: 89-92.

Peragine A, Yoshikawa M, Wu G, Albrecht HL, Poethig RS. 2004. SGS3 and SGS2/SDE1/RDR6 are required for juvenile development and the production of trans-acting siRNAs in Arabidopsis. Genes Dev 18: 2368-2379.

Schmutz J, Cannon SB, Schlueter J, Ma J, Mitros T, Nelson W, Hyten DL, Song Q, Thelen JJ, Cheng J, et al. 2010. Genome sequence of the palaeopolyploid soybean. Nature 463: 178183.

Soderlund C, Bomhoff M, Nelson WM. 2011. SyMAP v3. 4: A turnkey synteny system with application to plant genomes. Nucleic Acids Res 39: e68. doi: 10.1093/nar/gkr123.

Song QX, Liu YF, Hu XY, Zhang WK, Ma B, Chen SY, Zhang JS. 2011. Identification of miRNAs and their target genes in developing soybean seeds by deep sequencing. BMC Plant Biol 11: 5. doi: 10.1186/1471-2229-11-5.

Valdes-Lopez O, Yang SS, Aparicio-Fabre R, Graham PH, Reyes JL, Vance CP, Hernandez G. 2010. MicroRNA expression profile in common bean (Phaseolus vulgaris) under nutrient deficiency stresses and manganese toxicity. New Phytol 187: 805-818.

VandenBosch KA, Rodgers LR, Sherrier DJ, Kishinevsky BD. 1994. A peanut nodule lectin in infected cells and in vacuoles and the extracellular matrix of nodule parenchyma. Plant Physiol 104: 327-337.

Vaucheret H. 2006. Post-transcriptional small RNA pathways in plants: Mechanisms and regulations. Genes Dev 20: 759771.

Vaucheret H, Vazquez F, Crete P, Bartel DP. 2004. The action of ARGONAUTE1 in the miRNA pathway and its regulation by the miRNA pathway are crucial for plant development. Genes Dev 18: 1187-1197.

Vaucheret H, Mallory AC, Bartel DP. 2006. AGO1 homeostasis entails coexpression of MIR168 and AGO1 and preferential stabilization of miR168 by AGO1. Mol Cell 22: 129-136.

Wang JW, Czech B, Weigel D. 2009. miR156-regulated SPL transcription factors define an endogenous flowering pathway in Arabidopsis thaliana. Cell 138: 738-749.

Wu G, Park MY, Conway SR, Wang JW, Weigel D, Poethig RS. 2009. The sequential action of miR156 and miR 172 regulates developmental timing in Arabidopsis. Cell 138: 750-759.

Xie Z, Kasschau KD, Carrington JC. 2003. Negative feedback regulation of Dicer-Like1 in Arabidopsis by microRNAguided mRNA degradation. Curr Biol 13: 784-789.

Xu X, Pan S, Cheng S, Zhang B, Mu D, Ni P, Zhang G, Yang SPI, Li RPI, Wang JPI, et al. 2011. Genome sequence and analysis of the tuber crop potato. Nature 475: 189-195.

Yang S, Tang F, Gao M, Krishnan HB, Zhu H. 2010. R genecontrolled host specificity in the legume-rhizobia symbiosis. Proc Natl Acad Sci 107: 18735-18740.

Yoshikawa M, Peragine A, Park MY, Poethig RS. 2005. A pathway for the biogenesis of trans-acting siRNAs in Arabidopsis. Genes Dev 19: 2164-2175. 


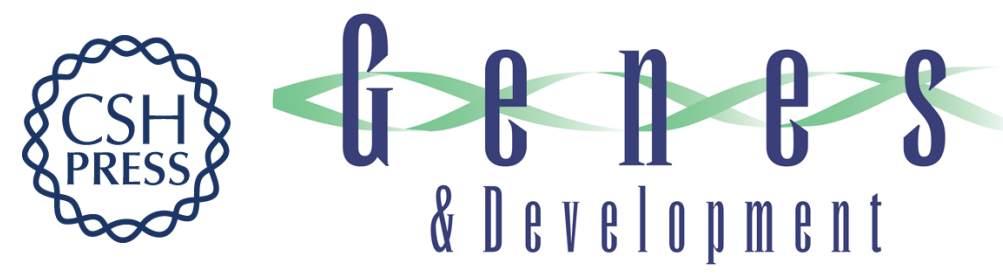

\section{MicroRNAs as master regulators of the plant $N B-L R R$ defense gene family via the production of phased, trans-acting siRNAs}

Jixian Zhai, Dong-Hoon Jeong, Emanuele De Paoli, et al.

Genes Dev. 2011, 25:

Access the most recent version at doi:10.1101/gad.177527.111

Supplemental http://genesdev.cshlp.org/content/suppl/2011/12/07/25.23.2540.DC1
Material

References This article cites 57 articles, 17 of which can be accessed free at:

http://genesdev.cshlp.org/content/25/23/2540.full.html\#ref-list-1

License

Email Alerting

Receive free email alerts when new articles cite this article - sign up in the box at the top

Service

right corner of the article or click here.

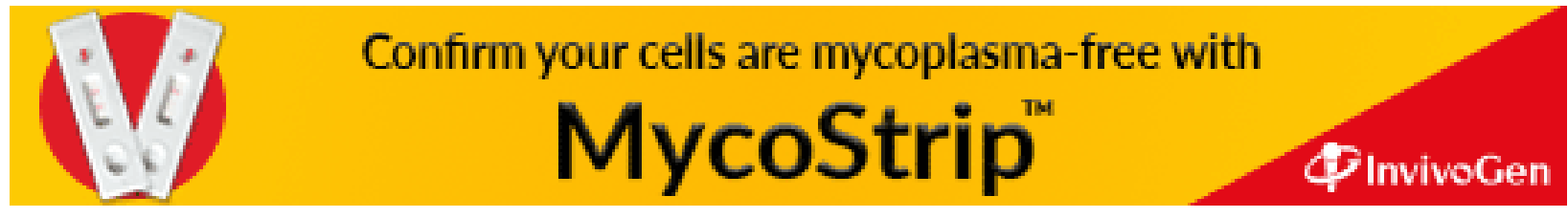

